\title{
Percent of Positive Nuclei
}

National Cancer Institute

\section{Source}

National Cancer Institute. Percent of Positive Nuclei. NCI Thesaurus. Code C159477.

A quantitative measurement of the percent of nuclei that are positive for a biomarker of interest compared to the total number of nuclei in a sample. 